Zur Darstellung von Umweltdaten in Nachhaltigkeitsberichten

\section{Von Emissionen, die sich in Luft auflösen}

Die Nachhaltigkeitsberichterstattung von Unternehmen hat sich in den letzten Jahren stetig weiterentwickelt. Leider gilt dieser positive Trend nicht für alle Bereiche der Berichterstattung. Bei der Darstellung von Umweltdaten weisen die Berichte der Unternehmen zum Teil gravierende Mängel auf. Von Andrea Liesen, Frank Figge, Tobias Hahn und Ralf Barkemeyer

$\mathbf{P}^{2}$ ositive Entwicklungen auf dem Gebiet der Nachhaltigkeitsberichterstattung von Unternehmen können beispielsweise durch die Analysen des IÖW/future-Rankings der Nachhaltigkeitsberichte (vgl. Beitrag von Gebauer in diesem Heft) belegt werden. Leider gilt dieser Fortschritt nicht gleichermaßen für die in den Berichten enthaltenen Daten zur Umweltbelastung. Diese weisen weiterhin starke Defizite auf. Ein bedauernswerter Zustand, denn ohne belastbare Umweltdaten lassen sich Unternehmen, die Fortschritte bei der Reduzierung ihrer Umweltbelastungen machen, nicht von jenen unterscheiden, die ihre Verantwortung der Umwelt gegenüber lediglich durch vage Aussagen bezeugen.

Vor diesem Hintergrund zeigen die langïhrigen Forschungsarbeiten der Sustainable Value Research Group die zum Teil gravierenden Missstände bei der Berichterstattung von Umweltdaten durch Unternehmen auf.

\section{Falsche Daten}

So berichten viele Unternehmen Umweltdaten nur für einen Teil ihrer Aktivitäten. Andere in den Nachhaltigkeitsund Umweltberichten veröffentlichte Daten sind schlichtweg falsch.

British Telecom berichtet beispielsweise, dass 99,8 Prozent des im internationalen Geschäft angefallenen Abfalls im Jahr 2008 von einer Handvoll Büromitarbeitern in Belgien produziert wurde, während Mitarbeiter auf dem australischen
Kontinent kein Wasser konsumiert haben. Der italienische Stromversorger ENEL hingegen berichtet im Nachhaltigkeitsbericht für das Jahr 2009 von rund 122 Milliarden Tonnen KohlenstoffdioxidEmissionen. Dies ist ein Vierfaches der durch den Menschen verursachten Emissionen weltweit und das 843-fache seines Wettbewerbers E.ON. E.ON wiederum hat gemeinsam mit Volkswagen einen eleganten legalen Weg gefunden ein ganzes Kraftwerk bilanziell verschwinden zu lassen - so lösen sich 2,5 Millionen Tonnen Kohlenstoffdioxid in Luft auf.

\section{Nur ausgewählte Aktivitäten}

Diese anekdotenhaften Beispiele sind jedoch nur ein Teil eines größeren Problems. Im Rahmen eines von der schwedischen MISTRA Stiftung geförderten Projekts analysierte Andrea Liesen von der Universität Leeds erstmals ausführlich die Qualität von Daten zur Treibhausgasberichterstattung von 431 Unternehmen über einen Zeitraum von fünf Jahren. Mit ernüchternden Ergebnissen: Im Durchschnitt berichteten in den Jahren 2005 bis 2009 lediglich ein Viertel der großen europäischen Aktiengesellschaften Emissionsdaten, die die gesamten Aktivitäten des Unternehmen hingegen abbilden. Die Mehrheit der Unternehmen berichtet Emissionen nur für einen Teil ihrer Aktivitäten, in Bezug auf bestimmte geografische Gebiete oder gibt die Grenzen ihrer Berichterstattung erst gar nicht an.
Dies überrascht, da sowohl das Greenhouse Gas Protocol als auch das Carbon Disclosure Project Unternehmen die Berichterstattung von direkten und indirekten Treibhausgasemissionen für die gesamten Aktivitäten des Unternehmens empfehlen. Der Empfehlung folgten im Durchschnitt lediglich 15 Prozent der berichtenden Unternehmen in den Jahren 2005 bis 2009. Dieser niedrige Wert verdeutlicht, dass eine einfache standardisierte Bewertung von Unternehmen auf Basis der berichteten Umweltdaten fast unmöglich ist. Auch wird deutlich, dass Initiativen wie das Greenhouse Gas Protocol Unternehmen zwar einen nützlichen Leitfaden für die Berichterstattung liefern, diese und andere Richtlinien jedoch ohne rechtliche Bindung nicht konsequent angewandt werden.

\section{Ein handfester Skandal?}

Ein Blick zur Finanzberichterstattung verdeutlicht das Problem. Würden Unternehmen in der Finanzberichterstattung nur über einen Teil ihrer Unternehmensaktivitäten berichten, wäre dies ein handfester Skandal. In der Nachhaltigkeitsberichterstattung ist es die gängige Praxis.

I AUTOREN + KONTAKT

Andrea Liesen forscht an der Universität Leeds, Großbritannien.

Frank Figge ist Professor für Sustainable Development und Corporate Sustainability an der Euromed Management School in Marseille, Frankreich.

Tobias Hahn ist Associate Professor für Corporate Sustainability, CSR and Environmental Management an der Euromed Management School in Marseille.

Ralf Barkemeyer ist Lecturer für Corporate Social Responsibility an der Universität Leeds.

Die Sustainable Value Research Group umfasst Forscher der Euromed Management School und der Universität Leeds.

E-Mail: info@sustainablevalue.com 


\section{Lizenzhinweis}

Die Beiträge in ÖkologischesWirtschaften werden unter der Creative-Commons-Lizenz "CC 4.0 Attribution Non-Commercial No Derivatives" veröffentlicht. Im Rahmen dieser Lizenz muss der Autor/Urheber stets genannt werden, das Werk darf nicht bearbeitet, abgewandelt oder in anderer Weise verändert und außerdem nicht kommerziell genutzt werden. Die digitale Version des Artikels bleibt für zwei Jahre Abonnent/innen vorbehalten und ist danach im Open Access verfügbar. 